\title{
Black Phosphorus Q-Switched Large-Mode-Area Tm-Doped Fiber Laser
}

\author{
Yan Ren, ${ }^{1}$ Zhipeng Qin, ${ }^{1}$ Guoqiang Xie ${ }^{\mathbb{D}},{ }^{1}$ Zhen Qiao, ${ }^{1}$ Jingui Ma, ${ }^{1}$ Peng Yuan, \\ Liejia Qian, ${ }^{1}$ Shikai Wang $\mathbb{D}^{2},{ }^{2}$ Chunlei $\mathrm{Yu}^{2}$, and Lili $\mathrm{Hu}^{2}$ \\ ${ }^{1}$ Key Laboratory for Laser Plasmas (Ministry of Education), Collaborative Innovation Center of IFSA (CICIFSA), \\ School of Physics and Astronomy, Shanghai Jiao Tong University, Shanghai 200240, China \\ ${ }^{2}$ Key Laboratory of High Power Laser Materials, Shanghai Institute of Optics and Fine Mechanics, Chinese Academy of Sciences, \\ Shanghai 201800, China
}

Correspondence should be addressed to Guoqiang Xie; xiegq@sjtu.edu.cn and Shikai Wang; woshiwsk@163.com

Received 5 August 2017; Revised 29 November 2017; Accepted 16 December 2017; Published 1 February 2018

Academic Editor: Giulio Cerullo

Copyright (C) 2018 Yan Ren et al. This is an open access article distributed under the Creative Commons Attribution License, which permits unrestricted use, distribution, and reproduction in any medium, provided the original work is properly cited.

We report on a passively Q-switched fiber laser with black phosphorus as saturable absorber. By employing the sol-gel fabricated large-mode-area Tm-doped fiber as gain medium, a high-energy Q-switched fiber laser has been demonstrated which delivers the maximum pulse energy of $11.72 \mu \mathrm{J}$ with the pulse width of $660 \mathrm{~ns}$ at the wavelength of $1954 \mathrm{~nm}$. Our experimental results indicate that BP Q-switched large-mode-area Tm-doped fiber laser is an effective and reliable approach to generate high-energy pulses at $2 \mu \mathrm{m}$.

\section{Introduction}

High-power and high-energy pulsed lasers operating at $2 \mu \mathrm{m}$ from the ${ }^{3} \mathrm{~F}_{4} \rightarrow{ }^{3} \mathrm{H}_{6}$ transition of $\mathrm{Tm}^{3+}$ ion have been extensively studied for their potential applications such as material processing, laser therapy, national defense security, freespace optical communication, and pumping for mid-infrared supercontinuum generation [1-5]. Passively Q-switched Tmdoped fiber lasers are favorable ways to generate high-energy pulses at $2 \mu \mathrm{m}$, where two-dimension (2D) materials such as graphene, topological insulators (TIs), and transition metal dichalcogenides (TMDCs) are usually adopted as saturable absorbers (SAs) instead of commercial semiconductor saturable absorber mirror (SESAM) due to their low cost and easy fabrication [6-13].

Black phosphorus (BP), as a newly emerged 2D material, has attracted wide attention recently for the common properties of 2D materials with wide bandwidth, ultrafast carrier dynamics, and planar characteristic [14]. More importantly, BP has a thickness-dependent direct energy bandgap from $0.3 \mathrm{eV}$ (bulk) to $2.0 \mathrm{eV}$ (single layer) [15], filling up the interval between the zero gap of graphene and large gap of TMDCs, which is of great importance to the optical applications. Up to now, the broadband BP saturable absorbers have been widely applied to pulsed lasers in a wide spectral range from visible to mid-infrared [16-25]. Specifically, in $2 \mu \mathrm{m}$ Q-switched fiber lasers, Wang et al. reported a passively Q-switched Tmdoped fiber laser using a BP deposited microfiber, delivering the maximum pulse energy of $154 \mathrm{~nJ}$ [26]; Jiang et al. deposited the BP powders onto a side-polished fiber and achieved Q-switched operation with the maximum pulse energy of $276 \mathrm{~nJ}$ [27]. The output pulse energy was further improved up to $632 \mathrm{~nJ}$ with BP SA fabricated by optical deposition method [28]. Although BP has been confirmed to be a reliable and excellent SA for $2.0 \mu \mathrm{m}$ Q-switched fiber lasers, the output pulse energy is limited below micro joule level due to the employment of single-mode fibers. Large-mode-area (LMA) fiber fabricated via sol-gel method combined with high temperature sintering will be a choice for generating high-energy laser pulses. The sol-gel method has a great merit of higher doping homogeneity for rareearth ions, preventing the cluster of the rare-earth ions and the fluorescence quench effect. With the advance of LMA fiber fabrication, dual-cladding Tm-doped fiber with core diameter as large as $38 \mu \mathrm{m}$ could be developed by sol-gel method combined with high temperature sintering [29], 


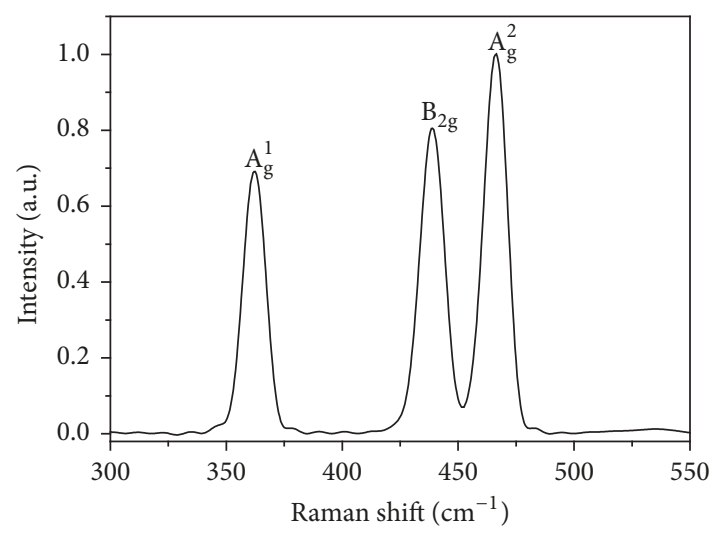

(a)

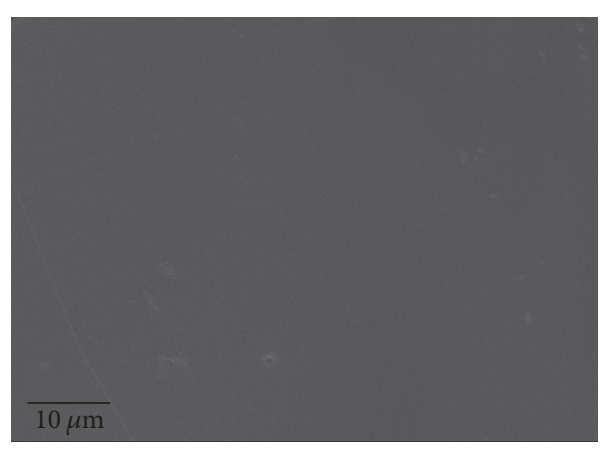

(b)

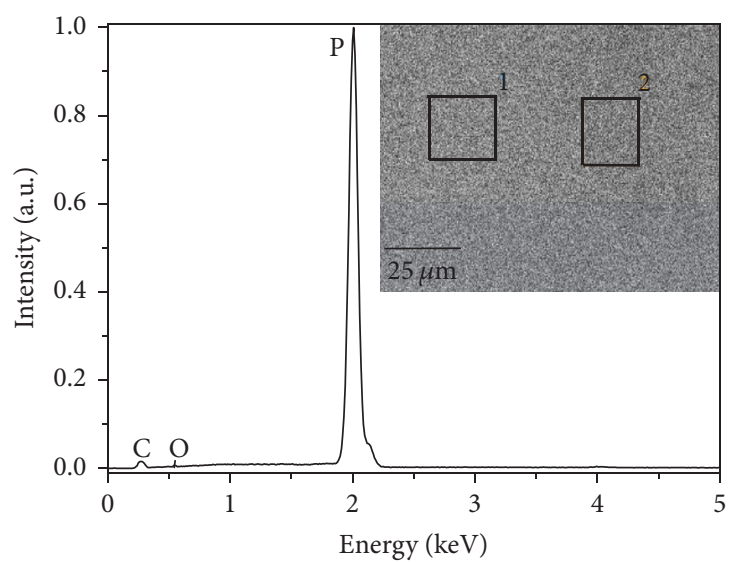

(d)

FIGURE 1: (a) Raman spectrum of the BP flake sample. (b) Morphology of BP flake measured by the scanning electron microscopy (SEM). (c) Three-dimension morphology of the BP flake scanned by the atomic force microscopy (AFM). Inset: height difference between mica sheet substrate (point A) and BP flake (point B). (d) Elemental components measured by the energy-dispersive X-ray spectroscopy (EDS). Inset: SEM image of BP flake.

offering a good alternative for higher pulse energy generation at $2 \mu \mathrm{m}$.

In this paper, we adopted the optimized LMA doublecladding Tm-doped fiber as gain medium with a core diameter as large as $30 \mu \mathrm{m}$. Based on a mechanically exfoliated BP SA, the high-energy Q-switching operation was demonstrated. The Q-switched fiber laser emitted pulses with the maximum average output power of $615 \mathrm{~mW}$, the maximum pulse energy of $11.72 \mu \mathrm{J}$, and the shortest pulse width of $660 \mathrm{~ns}$ at $1954 \mathrm{~nm}$. Compared with the previously reported pulse energies in BP Q-switched Tm-doped fiber lasers, the pulse energy was improved by an order of magnitude, indicating that the sol-gel fabricated LMA Tm-doped fiber together with BP SA is an effective way to generate Q-switched fiber laser with high pulse energy at the wavelength of $2 \mu \mathrm{m}$.

\section{Characteristics of BP Flake}

Similar to the preparation approach of graphene, the layered BP flake could also be prepared via mechanical exfoliation method. By repeatedly exfoliating a bulk BP with scotch tape, the thin BP flake could be obtained. As displayed in Figure 1, the characteristics of as-prepared BP sample were analyzed.
Excited by a $532 \mathrm{~nm}$ laser, Raman spectrum (Figure 1(a)) of the sample reveals three apparent peaks at the wavenumbers of $362 \mathrm{~cm}^{-1}, 439 \mathrm{~cm}^{-1}$, and $466 \mathrm{~cm}^{-1}$, corresponding to mode vibrations $\mathrm{A}_{\mathrm{g}}^{1}, \mathrm{~B}_{2 \mathrm{~g}}$, and $\mathrm{A}_{\mathrm{g}}^{2}$ of phosphorus atoms in BP crystal lattice, respectively [30-32]. Using the scanning electron microscopy (SEM), the morphology of the as-prepared BP flake was obtained with an amplification rate of 1000 . As Figure 1(b) shows, the smooth surface indicated that the mechanically exfoliated BP flake is uniform with a relatively high quality. To further confirm the thickness of the BP flake, the thickness measurement was performed with the atomic force microscopy (AFM). The three-dimensional (3D) AFM image (Figure 1(c)) shows the existence of some bubble-like bulges on the surface. The inset of Figure 1(c) shows that the height difference between the mica sheet substrate (point A) and the BP flake (point B) is $7.0 \mu \mathrm{m}$. Since the thickness of monolayer BP is $0.6 \mathrm{~nm}$, the as-prepared BP flake could be regarded as bulk-like form with an energy gap of $0.3 \mathrm{eV}$, supporting the saturable absorption up to $4.1 \mu \mathrm{m}$ wavelength. The elemental components of BP flake were confirmed by energy-dispersive $\mathrm{X}$-ray spectroscopy (EDS). The analysis of the spectroscopic data in Figure 1(d) demonstrates the existence of carbon $(\mathrm{C})$, oxygen $(\mathrm{O})$, and phosphorus $(\mathrm{P})$. 


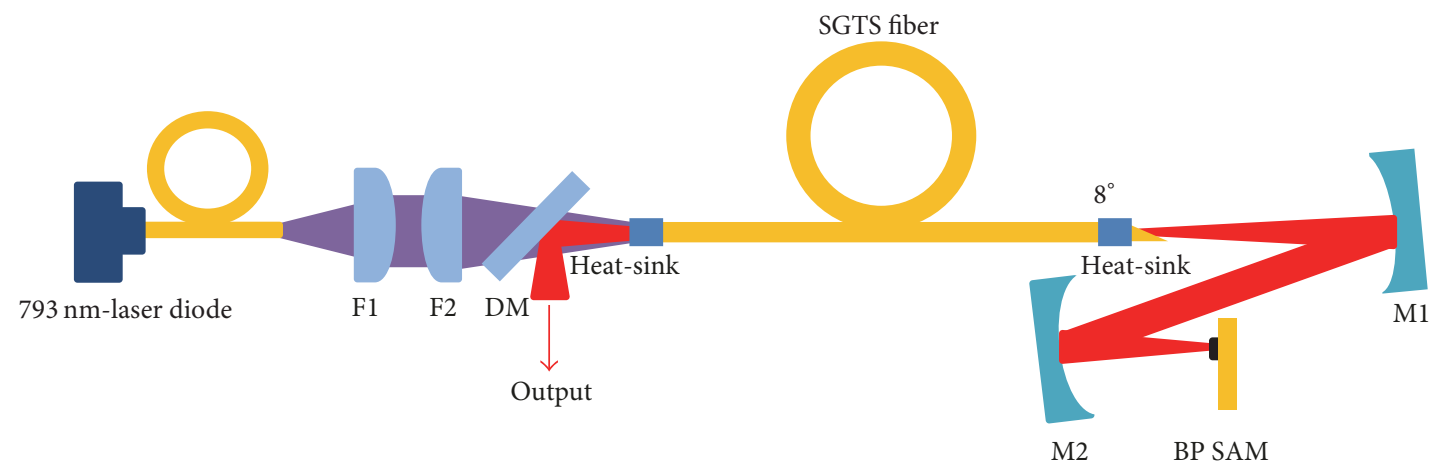

FIGURE 2: Experimental setup schematic of the BP Q-switched LMA Tm-doped fiber laser. DM: dichroic mirror; LMA TDF: large-mode-area Tm-doped fiber; BP SAM: black phosphorus saturable absorber mirror.

Two different rectangle areas with a size of about $400 \mu \mathrm{m}^{2}$ (inset of Figure 1(d)) were chosen for statistics. The average percentages of each component are $93.7 \%, 1.3 \%$, and $5 \%$ in weight for $\mathrm{P}, \mathrm{O}$, and $\mathrm{C}$, respectively. The existence of $\mathrm{C}$ and $\mathrm{O}$ might be attributed to defect in the fabrication process of bulk BP and oxidation in the atmosphere, respectively. The asprepared BP flake was transferred onto a gold-coated mirror to fabricate a BP saturable absorber mirror (SAM). Compared with the BP dispersed in chemical solvent or polished into powder, the mechanically exfoliated BP flake is of high quality with flat surface, high purity, and large size which are very suitable to LMA Tm-doped fiber lasers for high-energy pulse generation.

\section{Experimental Setup}

The experimental setup schematic of BP passively Q-switched LMA Tm-doped fiber laser is depicted in Figure 2. The fibercoupled $793 \mathrm{~nm}$ laser diode (LD) with a core diameter of $105 \mu \mathrm{m}$ and a numerical aperture of 0.15 was adopted as the pump source. The pump laser from the LD was collimated and then focused onto the gain fiber by a pair of planoconvex lenses $\mathrm{F} 1$ and $\mathrm{F} 2\left(f_{1}=f_{2}=75 \mathrm{~mm}\right)$. The $4 \mathrm{~m}$ length LMA double-cladding Tm-doped fiber was adopted as the gain fiber. The double-cladding Tm-doped fiber has a glass composition of $0.1 \mathrm{Tm}_{2} \mathrm{O}_{3}-1.5 \mathrm{Al}_{2} \mathrm{O}_{3}-98.4 \mathrm{SiO}_{2}$ in mol.\% and a large core diameter of $30 \mu \mathrm{m}$ with a NA of 0.102 . The first cladding diameter and NA of the gain fiber were $250 \mu \mathrm{m}$ and 0.366 , respectively, guaranteeing the effective pump coupling. The normalized frequency of the fiber is about 4.9 for $1954 \mathrm{~nm}$, indicating that the gain fiber supports multimode operation. In order to generate high-quality beam, the gain fiber was rolled up with a folding diameter of $\sim 15 \mathrm{~cm}$ to suppress high-order transverse mode oscillation. The input fiber end was cleaved perpendicularly and acted as cavity feedback with Fresnel reflectivity of $4 \%$ and output coupler with a transmissivity of $96 \%$. The other fiber end was cut with an angle of $8^{\circ}$ to avoid parasitic oscillation. In order to remove the heat while pumping, both ends of fiber were mounted in an aluminum heat sink with a $\mathrm{V}$-groove. The intracavity laser beam was collimated and focused onto BP SAM by a pair of concave mirrors $\mathrm{M} 1$ and $\mathrm{M} 2$ with a radius of curvature (ROC) of $150 \mathrm{~mm}$ and $100 \mathrm{~mm}$, respectively. The residual pump light was filtered by the two concave mirrors (M1

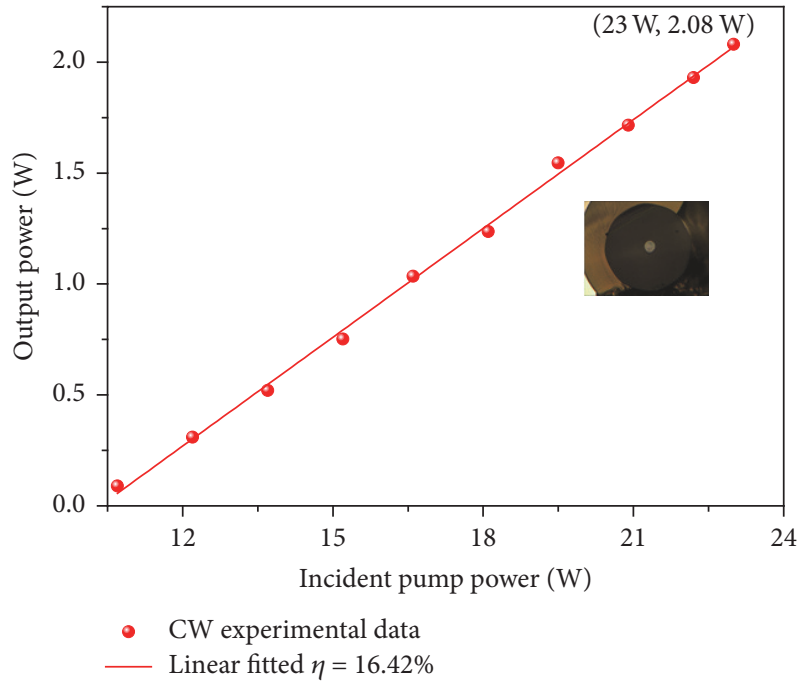

FIGURE 3: Continuous-wave output powers versus launched pump powers.

and M2) with antireflection coating for pump wavelength, avoiding the adverse effect such as additional heat load on BP SAM. In order to separate the output laser beam from the pump beam, the $45^{\circ}$-placed dichroic mirror (DM) was coated with high transmissivity for pump wavelength $(T>$ $95 \%)$ and high reflectivity for laser wavelength $(R>99 \%)$. For the convenience of measurement, the output laser beam was collimated by a plano-convex $\mathrm{CaF}_{2}$ lens.

\section{Results and Discussion}

Using a highly reflective mirror instead of BP SAM, the continuous-wave (CW) laser performances with differentlength Tm-doped fibers were tested and $4 \mathrm{~m}$ length LMA Tmdoped fiber demonstrated the best laser performance. CW operation with $4 \mathrm{~m}$ length gain fiber was achieved with a threshold of 10.7 W, as shown in Figure 3. With the increase of the launched pump power, the CW output power increased linearly with a slope efficiency of $16.42 \%$ and the maximum output power of $2.08 \mathrm{~W}$ was obtained at the launched pump power of $23 \mathrm{~W}$. The output beam quality was measured by a commercial beam profiler (Thorlabs, M2MS, 400-2700 nm) with a $M^{2}$ factor of 1.3 [33]. Higher output power was only 


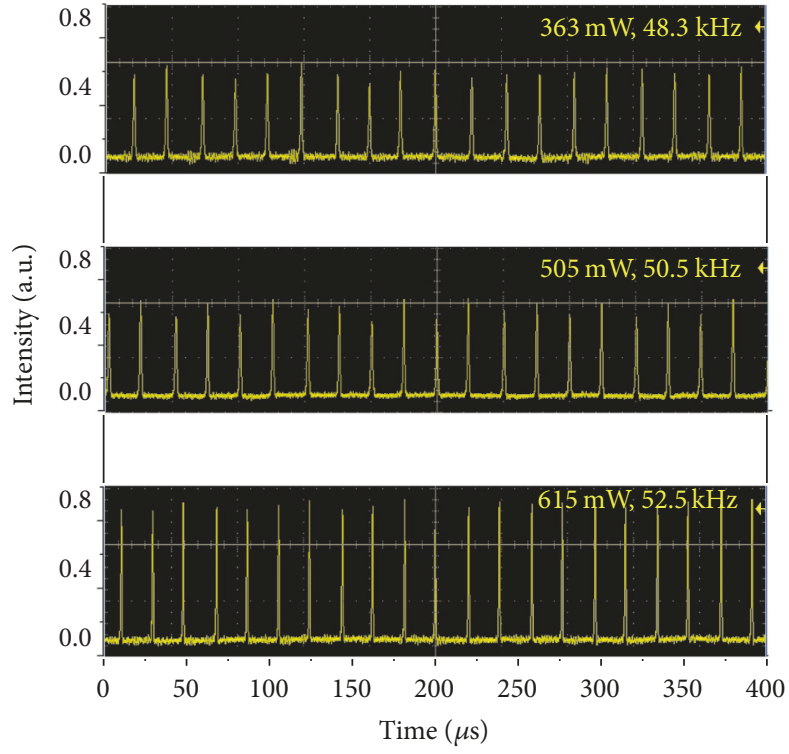

(a)

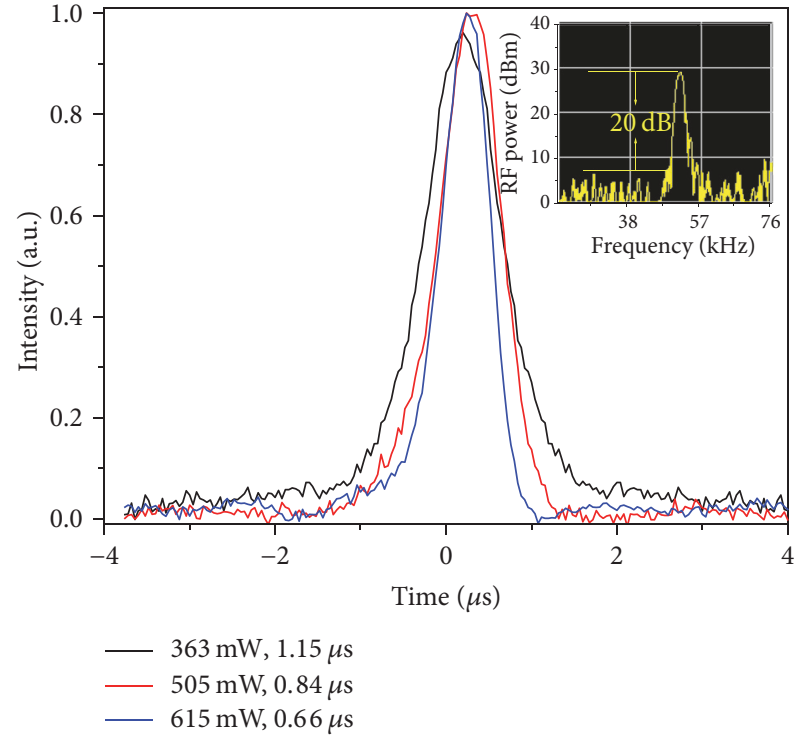

(b)

Figure 4: (a) Typical pulse trains at the average output power of $363 \mathrm{~mW}, 505 \mathrm{~mW}$, and $615 \mathrm{~mW}$, respectively. (b) Corresponding pulse profiles. Inset: RF spectrum recorded at the average output power of $615 \mathrm{~mW}$.

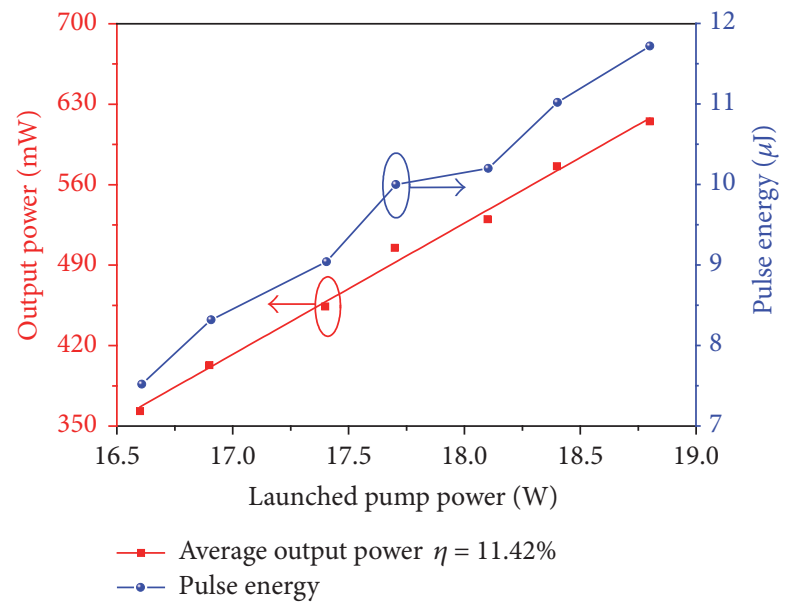

(a)

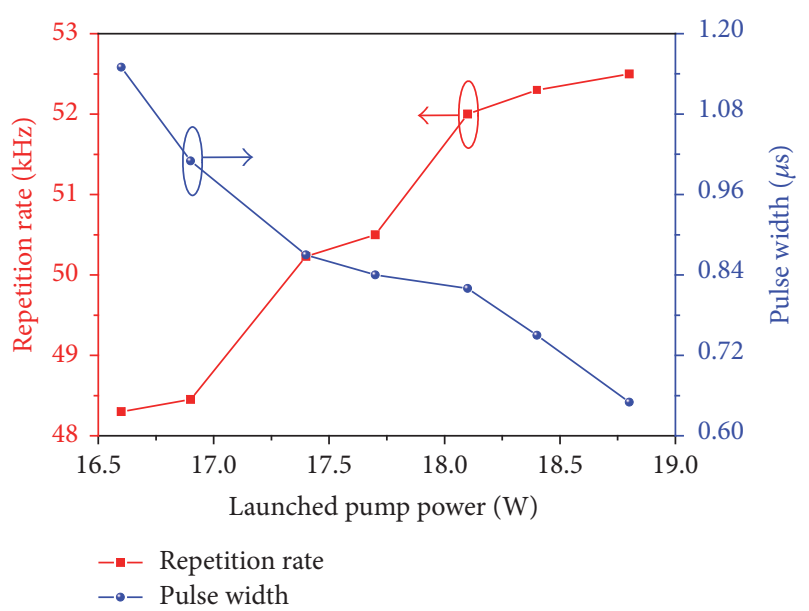

(b)

FiguRE 5: (a) Average output power and pulse energy versus the launched pump power. (b) Repetition rate and pulse width versus the launched pump power.

limited by the available pump power in this experiment. In the whole pump power range from laser threshold to the maximum pump power, we did not observe the self-pulsed phenomenon. The inset of Figure 3 shows the cross section of the gain fiber. In order to achieve Q-switching operation, we replaced the highly reflective mirror with BP SAM.

By precisely adjusting the longitudinal and transverse position of BP SAM, self-started Q-switching operation was observed on a digital oscilloscope at the launched pump power of 16.6 W. Figure 4(a) shows three typical Q-switched pulse trains recorded at the output power of $363 \mathrm{~mW}, 505 \mathrm{~mW}$, and $615 \mathrm{~mW}$, respectively. Each output power corresponded to a repetition rate which increased with the launched pump power. The corresponding pulse profiles are shown in Figure 4(b), which were captured by a photoelectric detector (ALPHALAS, UPD-5N-IR2-P). The radio frequency (RF) spectrum (inset of Figure 4(b)), recorded at the average output power of $615 \mathrm{~mW}$, shows a signal-to-noise ratio of $20 \mathrm{~dB}$.

Figure 5(a) shows the average output power and pulse energy variation with the launched pump power in the passively Q-switched LMA Tm-doped fiber laser. Both average 
TABLE 1: Passively Q-switched Tm-doped and Tm/Ho-co doped fiber lasers based on low dimensional SAs.

\begin{tabular}{|c|c|c|c|c|c|c|c|}
\hline SA & $\begin{array}{l}\text { Core diameter } \\
\qquad(\mu \mathrm{m})\end{array}$ & $\begin{array}{c}\text { Average power } \\
(\mathrm{mW})\end{array}$ & $\begin{array}{l}\text { Wavelength } \\
(\mathrm{nm})\end{array}$ & $\begin{array}{c}\text { Pulse width } \\
(\mu \mathrm{s})\end{array}$ & $\begin{array}{c}\text { Pulse energy } \\
(\mu \mathrm{J})\end{array}$ & $\begin{array}{l}\text { Peak power } \\
\text { (W) }\end{array}$ & Ref. \\
\hline CNT & 9 & 1.2 & 1890 & 7.2 & $8.6 \times 10^{-2}$ & 0.01 & [11] \\
\hline Graphene & 6 & 4.5 & 2000 & 1.4 & $8.5 \times 10^{-2}$ & 0.06 & {$[12]$} \\
\hline Graphene & 6 & 96 & 1957 & 0.76 & 0.38 & 0.5 & {$[8]$} \\
\hline Graphene & 10 & 300 & 2032 & 3.8 & 6.7 & 1.8 & [13] \\
\hline Graphene & 10 & 5200 & 2005 & 0.32 & 18 & 56 & [34] \\
\hline $\mathrm{Bi}_{2} \mathrm{Se}_{3}$ & 9 & 8.4 & 1980 & 4.2 & 0.31 & 0.07 & [9] \\
\hline $\mathrm{Bi}_{2} \mathrm{Te}_{3}$ & 9 & 0.68 & 1890 & 1.7 & $1.2 \times 10^{-2}$ & $6.7 \times 10^{-3}$ & [10] \\
\hline $\mathrm{MoS}_{2}$ & 10 & 47 & 2032 & 1.8 & 1.0 & 0.56 & {$[6]$} \\
\hline $\mathrm{MoSe}_{2}$ & - & 1.0 & 1924 & 5.5 & $4.2 \times 10^{-2}$ & $7.6 \times 10^{-3}$ & [7] \\
\hline $\mathrm{BP}$ & 9 & - & 1890 & 2.5 & 0.28 & 0.1 & [27] \\
\hline $\mathrm{BP}$ & 10 & 3.8 & 1948 & 5.6 & 0.15 & 0.27 & [26] \\
\hline $\mathrm{BP}$ & 10 & 72 & 1912 & 0.73 & 0.63 & 0.86 & [28] \\
\hline $\mathrm{BP}$ & 30 & 615 & 1954 & 0.66 & 11.72 & 18 & $\begin{array}{l}\text { This } \\
\text { work }\end{array}$ \\
\hline
\end{tabular}

output power and pulse energy increased linearly with the launched pump power in the Q-switched regime. When the pump power increased to $18.8 \mathrm{~W}$, the maximum pulse energy of $11.72 \mu \mathrm{J}$ was achieved. Compared with the previous reports of $2 \mathrm{D}$ SAs Q-switched fiber lasers at $2 \mu \mathrm{m}$ (Table 1), there was a significant improvement of pulse energy in our experiment due to the employment of LMA fiber. With further increasing the pump power beyond $18.8 \mathrm{~W}$, the pulse train of the passively Q-switched laser became unstable and then disappeared. This phenomenon might be attributed to performance degradation of BP flake due to excess heat. With the advance of fiber fabrication and optimization of BP SAM, we believe that higher pulse energy will be achieved.

Figure 5(b) shows the evolution of repetition rate and pulse width with the launched pump power. As expected, with the increase of launched pump power from $16.6 \mathrm{~W}$ to $18.8 \mathrm{~W}$, the repetition rate increased from $48.3 \mathrm{kHz}$ to $52.5 \mathrm{kHz}$ and the pulse width decreased from $1.15 \mu \mathrm{s}$ to $0.66 \mu$ s, respectively. During the experiment, no Q-switching mode-locked pulses were obtained.

At the maximum average output power of $615 \mathrm{~mW}$, the spectrum of Q-switched fiber laser was measured by a mid-infrared spectrum analyzer with a resolution of $0.2 \mathrm{~nm}$ (Ocean Optics, SIR5000). As depicted in Figure 6, the Qswitched spectrum centered at the wavelength of $1954 \mathrm{~nm}$ with a full width of half maximum (FWHM) of $3.2 \mathrm{~nm}$.

\section{Conclusions}

In conclusion, we have experimentally demonstrated a BP passively Q-switched fiber laser with LMA double-cladding Tm-doped fiber as gain medium for the first time. The Qswitched fiber laser emitted pulses with a maximum pulse energy of $11.72 \mu \mathrm{J}$ and the shortest pulse width of $0.66 \mu \mathrm{s}$ at the wavelength of $1954 \mathrm{~nm}$. The experimental results suggest that the sol-gel fabricated LMA dual-cladding Tm-doped fiber combined with BP SA is an effective way for generating highenergy laser pulses at the eye-safe wavelength of $2 \mu \mathrm{m}$.

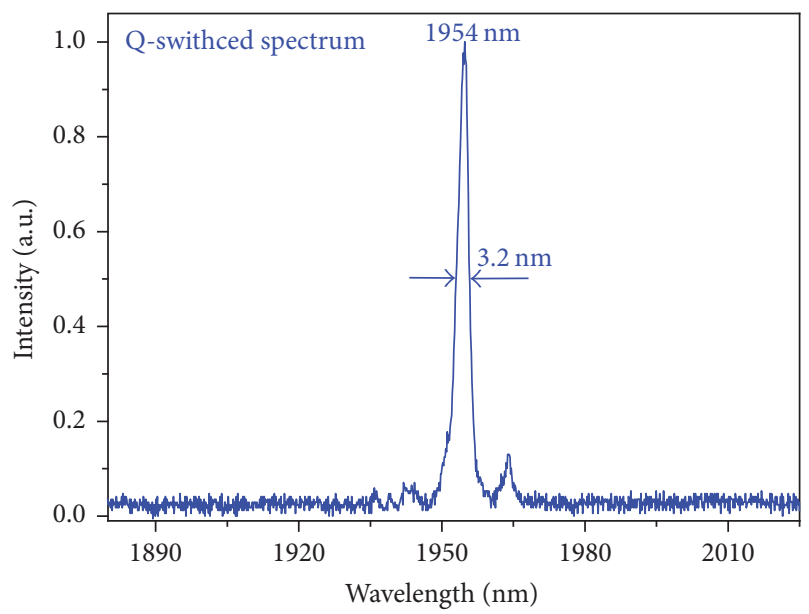

Figure 6: Q-switched pulse spectrum.

\section{Conflicts of Interest}

The authors declare that there are no conflicts of interest regarding the publication of this article.

\section{Acknowledgments}

The work is partially supported by the National Basic Research Program of China (Grant no. 2013CBA01505), the Shanghai Excellent Academic Leader Project (Grant no. 15XD1502100), and the National Natural Science Foundation of China (Grant nos. 61675130 and 11421064).

\section{References}

[1] T. Brabec and F. Krausz, "Intense few-cycle laser fields: frontiers of nonlinear optics," Reviews of Modern Physics, vol. 72, no. 2, pp. 545-591, 2000.

[2] W. Zeller, L. Naehle, P. Fuchs, F. Gerschuetz, L. Hildebrandt, and J. Koeth, "DFB lasers between $760 \mathrm{~nm}$ and $16 \mu \mathrm{m}$ for sensing applications," Sensors, vol. 10, no. 4, pp. 2492-2510, 2010. 
[3] C. R. Phillips, C. Langrock, J. S. Pelc et al., "Supercontinuum generation in quasi-phase-matched $\mathrm{LiNbO}_{3}$ waveguide pumped by a Tm-doped fiber laser system," Optics Letters, vol. 36, no. 19, pp. 3912-3914, 2011.

[4] N. M. Fried and K. E. Murray, "High-power thulium fiber laser ablation of urinary tissues at $1.94 \mu \mathrm{m}$," Journal of Endourology, vol. 19, no. 1, pp. 25-31, 2005.

[5] H. Chen, S.-P. Chen, Z.-F. Jiang, and J. Hou, "Diversified pulse generation from frequency shifted feedback Tm-doped fibre lasers," Scientific Reports, vol. 6, Article ID 26431, 2016.

[6] Z. Luo, Y. Huang, M. Zhong et al., "1-, 1.5-, and 2- $\mu \mathrm{m}$ fiber lasers Q-switched by a broadband few-layer $\mathrm{MoS}_{2}$ saturable absorber," Journal of Lightwave Technology, vol. 32, no. 24, Article ID 6920007, pp. 4077-4084, 2014.

[7] R. I. Woodward, R. C. T. Howe, T. H. Runcorn et al., "Wideband saturable absorption in few-layer molybdenum diselenide $\left(\mathrm{MoSe}_{2}\right)$ for Q-switching Yb-, Er- and Tm-doped fiber lasers," Optics Express, vol. 23, no. 15, pp. 20051-20061, 2015.

[8] M. Jiang, H. F. Ma, Z. Y. Ren et al., "A graphene Q-switched nanosecond Tm-doped fiber laser at $2 \mu \mathrm{m}$," Laser Physics Letters, vol. 10, no. 5, Article ID 055103, 2013.

[9] Z. Luo, C. Liu, Y. Huang et al., "Topological-insulator passively Q-switched double-clad fiber laser at $2 \mu \mathrm{m}$ wavelength," IEEE Journal of Selected Topics in Quantum Electronics, vol. 20, no. 5, Article ID 0902708, 2014.

[10] J. Lee, M. Jung, J. Koo, C. Chi, and J. H. Lee, "Passively Qswitched $1.89-\mu \mathrm{m}$ fiber laser using a bulk-structured $\mathrm{Bi}_{2} \mathrm{Te}_{3}$ topological insulator," IEEE Journal of Selected Topics in Quantum Electronics, vol. 21, no. 1, Article ID 0900206, 2015.

[11] M. Jung, J. Koo, Y. M. Chang, P. Debnath, Y.-W. Song, and J. H. Lee, "An all fiberized, 1.89- $\mu \mathrm{m}$ Q-switched laser employing carbon nanotube evanescent field interaction," Laser Physics Letters, vol. 9, no. 9, pp. 669-673, 2012.

[12] J. Liu, J. Xu, and P. Wang, "Graphene-based passively Qswitched $2 \mu \mathrm{m}$ thulium-doped fiber laser," Optics Communications, vol. 285, no. 24, pp. 5319-5322, 2012.

[13] C. Liu, C. Ye, Z. Luo et al., "High-energy passively Q-switched 2 $\mu \mathrm{m} \mathrm{Tm}^{3+}$ - doped double-clad fiber laser using grapheneoxidedeposited fiber taper," Optics Express, vol. 21, no. 1, pp. 204-209, 2013.

[14] J. Qiao, X. Kong, Z.-X. Hu, F. Yang, and W. Ji, "High-mobility transport anisotropy and linear dichroism in few-layer black phosphorus," Nature Communications, vol. 5, article no. 4475, 2014.

[15] V. Tran, R. Soklaski, Y. Liang, and L. Yang, "Layer-controlled band gap and anisotropic excitons in few-layer black phosphorus," Physical Review B: Condensed Matter and Materials Physics, vol. 89, no. 23, Article ID 235319, 2014.

[16] Y. Xie, L. Kong, Z. Qin, G. Xie, and J. Zhang, "Black phosphorusbased saturable absorber for Q-switched Tm: YAG ceramic laser," Optical Engineering, vol. 55, no. 8, Article ID 081307, 2016.

[17] J. Ma, S. Lu, Z. Guo et al., "Few-layer black phosphorus based saturable absorber mirror for pulsed solid-state lasers," Optics Express, vol. 23, no. 17, 2015.

[18] S. Zhang, X. Zhang, H. Wang et al., "Size-dependent saturable absorption and mode-locking of dispersed black phosphorus nanosheets," Optical Materials Express, vol. 6, no. 10, pp. 31593168, 2016.

[19] B. Zhang, F. Lou, R. Zhao et al., "Exfoliated layers of black phosphorus as saturable absorber for ultrafast solid-state laser," Optics Expresss, vol. 40, no. 16, pp. 3691-3694, 2015.
[20] Z. Qin, G. Xie, C. Zhao, S. Wen, P. Yuan, and L. Qian, "Midinfrared mode-locked pulse generation with multilayer black phosphorus as saturable absorber," Optics Letters, vol. 41, no. 1, pp. 56-59, 2016.

[21] Z. Qin, G. Xie, H. Zhang et al., "Black phosphorus as saturable absorber for the Q-switched Er: ZBLAN fiber laser at $2.8 \mu \mathrm{m}$," Optics Express, vol. 23, no. 19, pp. 24713-24718, 2015.

[22] J. Li, H. Luo, B. Zhai et al., "Black phosphorus: a two-dimension saturable absorption material for mid-infrared Q-switched and mode-locked fiber lasers," Scientific Reports, vol. 6, Article ID 30361, 2016.

[23] L. Kong, Z. Qin, G. Xie et al., "Black phosphorus as broadband saturable absorber for pulsed lasers from $1 \mu \mathrm{m}$ to $2.7 \mu \mathrm{m}$ wavelength," Laser Physics Letters, vol. 13, no. 4, Article ID 045801, 2016.

[24] J. Sotor, G. Sobon, W. Macherzynski, P. Paletko, and K. M. Abramski, "Black phosphorus saturable absorber for ultrashort pulse generation," Applied Physics Letters, vol. 107, no. 5, Article ID 051108, 2015.

[25] J. Sotor, G. Sobon, M. Kowalczyk, W. Macherzynski, P. Paletko, and K. M. Abramski, "Ultrafast thulium-doped fiber laser mode locked with black phosphorus," Optics Letters, vol. 40, no. 16, pp. 3885-3888, 2015.

[26] Y. Wang, J. Li, L. Han et al., "Q-switched $\mathrm{Tm}^{3+}$-doped fiber laser with a micro-fiber based black phosphorus saturable absorber," Laser Physics, vol. 26, no. 6, Article ID 065104, 2016.

[27] T. Jiang, K. Yin, X. Zheng, H. Yu, and X.-A. Cheng, Black Phosphorus as A New Broadband Saturable Absorber for Infrared Passively Q-Switched Fiber Lasers, 2015.

[28] H. Yu, X. Zheng, K. Yin, X. Cheng, and T. Jiang, "Nanosecond passively Q-switched thulium/holmium-doped fiber laser based on black phosphorus nanoplatelets," Optical Materials Express, vol. 6, no. 2, pp. 603-609, 2016.

[29] F. G. Lou, P. W. Kuan, L. Zhang et al., " $2 \mu \mathrm{m}$ laser properties of $\mathrm{Tm}^{3+}$-doped large core sol-gel silica fiber," Optical Materials Express, vol. 4, no. 6, pp. 1267-1275, 2014.

[30] H. Liu, A. T. Neal, Z. Zhu et al., "Phosphorene: an unexplored 2D semiconductor with a high hole mobility," ACS Nano, vol. 8, no. 4, pp. 4033-4041, 2014.

[31] Y. Akahama, M. Kobayashi, and H. Kawamura, "Raman study of black phosphorus up to $13 \mathrm{GPa}$," Solid State Communications, vol. 104, no. 6, pp. 311-315, 1997.

[32] Z.-C. Luo, M. Liu, Z.-N. Guo et al., "Microfiber-based few-layer black phosphorus saturable absorber for ultra-fast fiber laser," Optics Express, vol. 23, no. 15, pp. 20030-20039, 2015.

[33] Y. Ren, Z. P. Qin, and G. Q. Xie, 2- $\mu \mathrm{m}$ mode-locking laser performances of sol-gel-fabricated large-core Tm-doped silica fiber, Chinese Optics Letter.

[34] Y. Tang, X. Yu, X. Li, Z. Yan, and Q. J. Wang, "High-power thulium fiber laser Q-switched with single-layer graphene," Optics Letters, vol. 39, no. 3, pp. 614-617, 2014. 

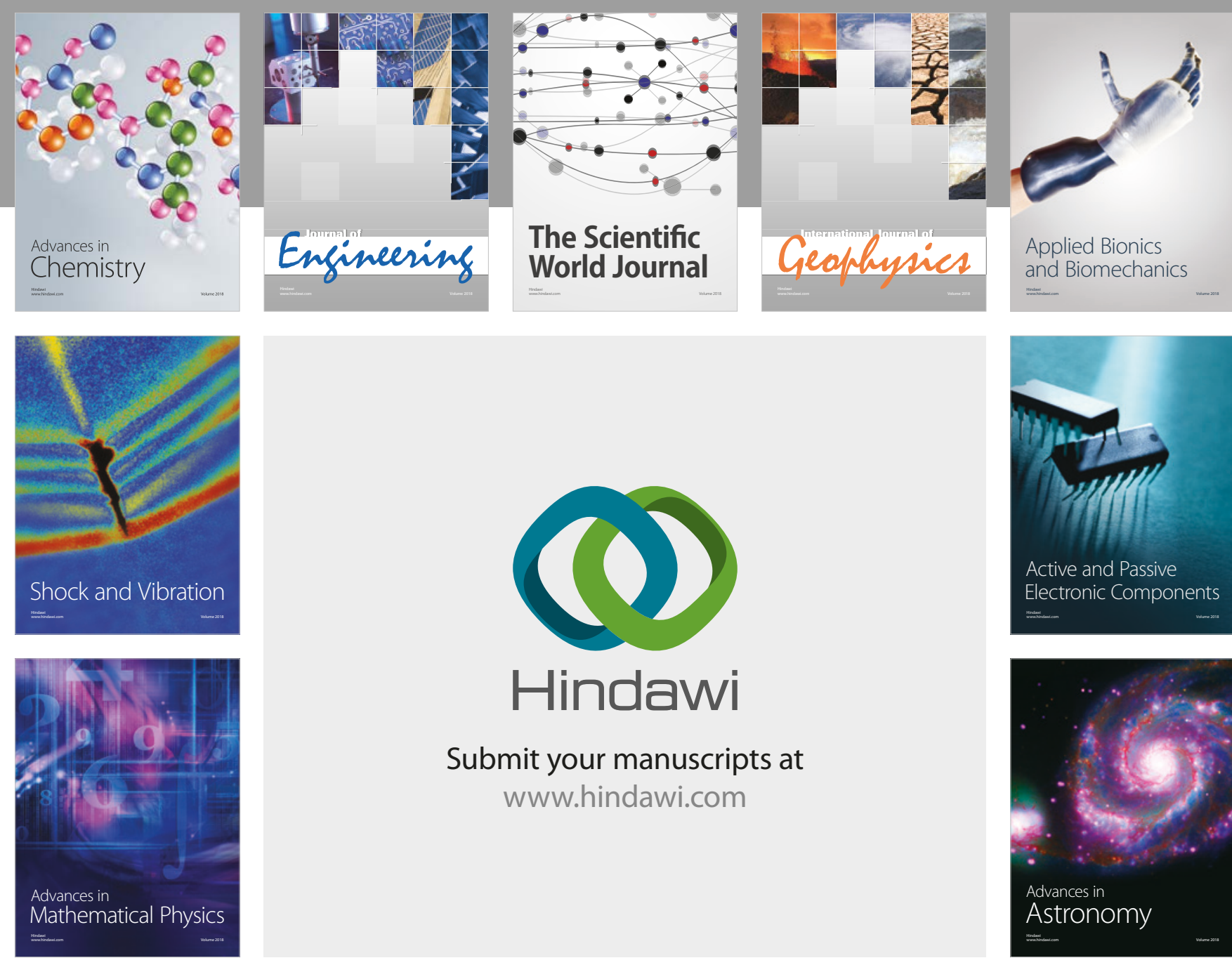

Submit your manuscripts at

www.hindawi.com

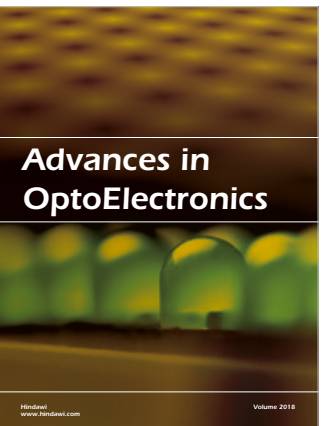

\section{Rotcting Machinery}
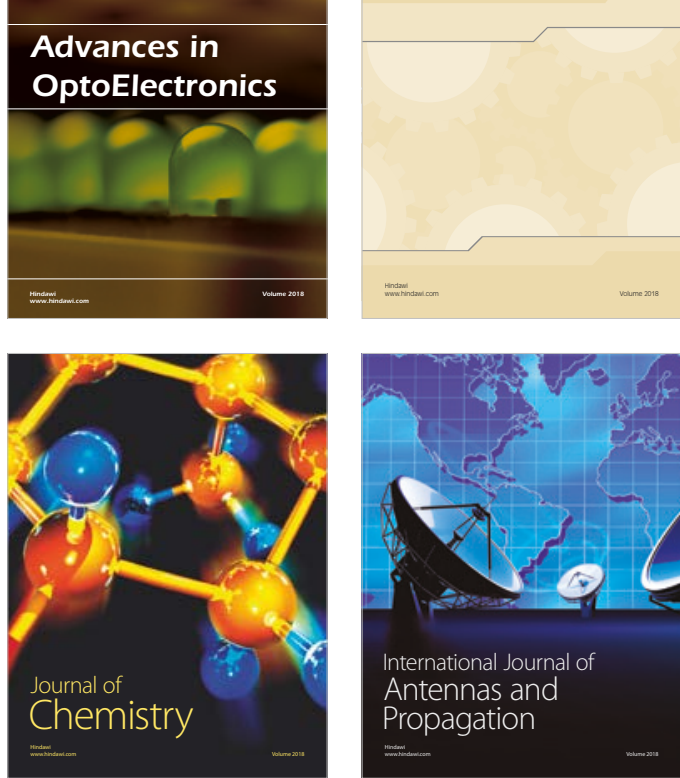

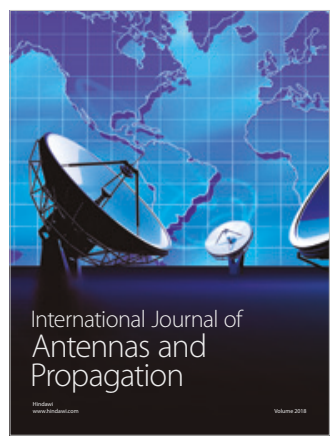

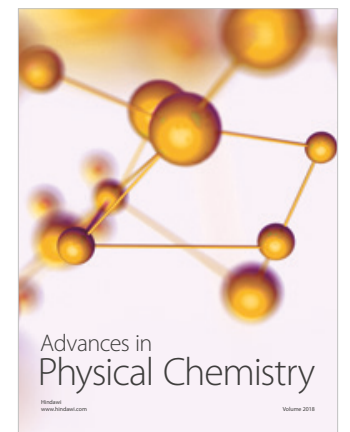

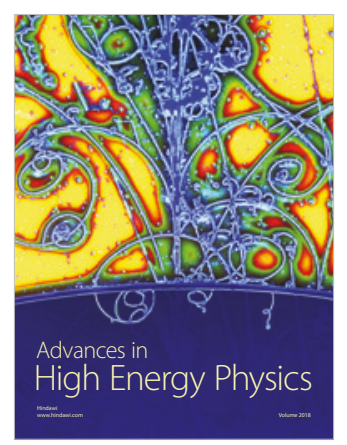

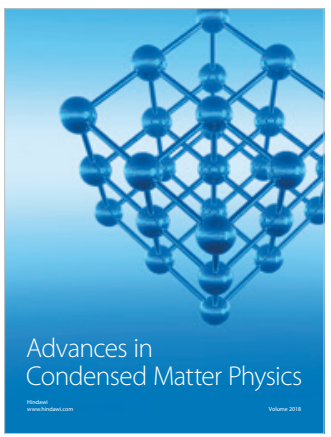

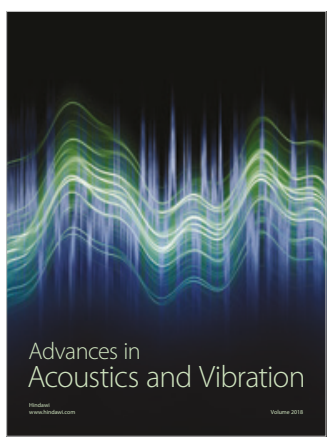

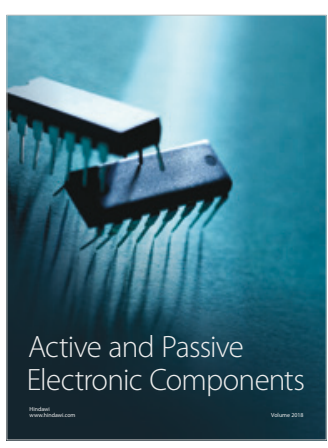
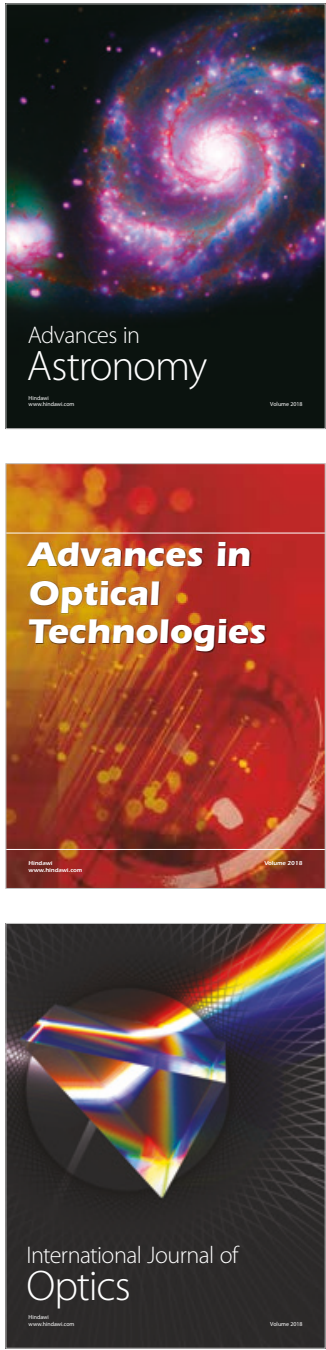